\title{
Du combat
}

Jouer et faire la guerre en Afrique (post-)coloniale et contemporaine About Combat. Playing and Making War in (Post)Colonial and Contemporary Africa

Thomas Riot, Nicolas Bancel et Herrade Boistelle

\section{(2) OpenEdition Journals}

Édition électronique

URL : https://journals.openedition.org/tc/8374

DOI : $10.4000 /$ tc. 8374

ISSN : 1952-420X

Éditeur

Éditions de l'EHESS

\section{Édition imprimée}

Date de publication : 1 décembre 2014

Pagination : 150-165

ISBN : 978-2-7351-2346-9

ISSN : 0248-6016

Référence électronique

Thomas Riot, Nicolas Bancel et Herrade Boistelle, « Du combat », Techniques \& Culture [En ligne], 62 | 2014, mis en ligne le 01 décembre 2017, consulté le 03 octobre 2022. URL : http:// journals.openedition.org/tc/8374; DOI : https://doi.org/10.4000/tc.8374 


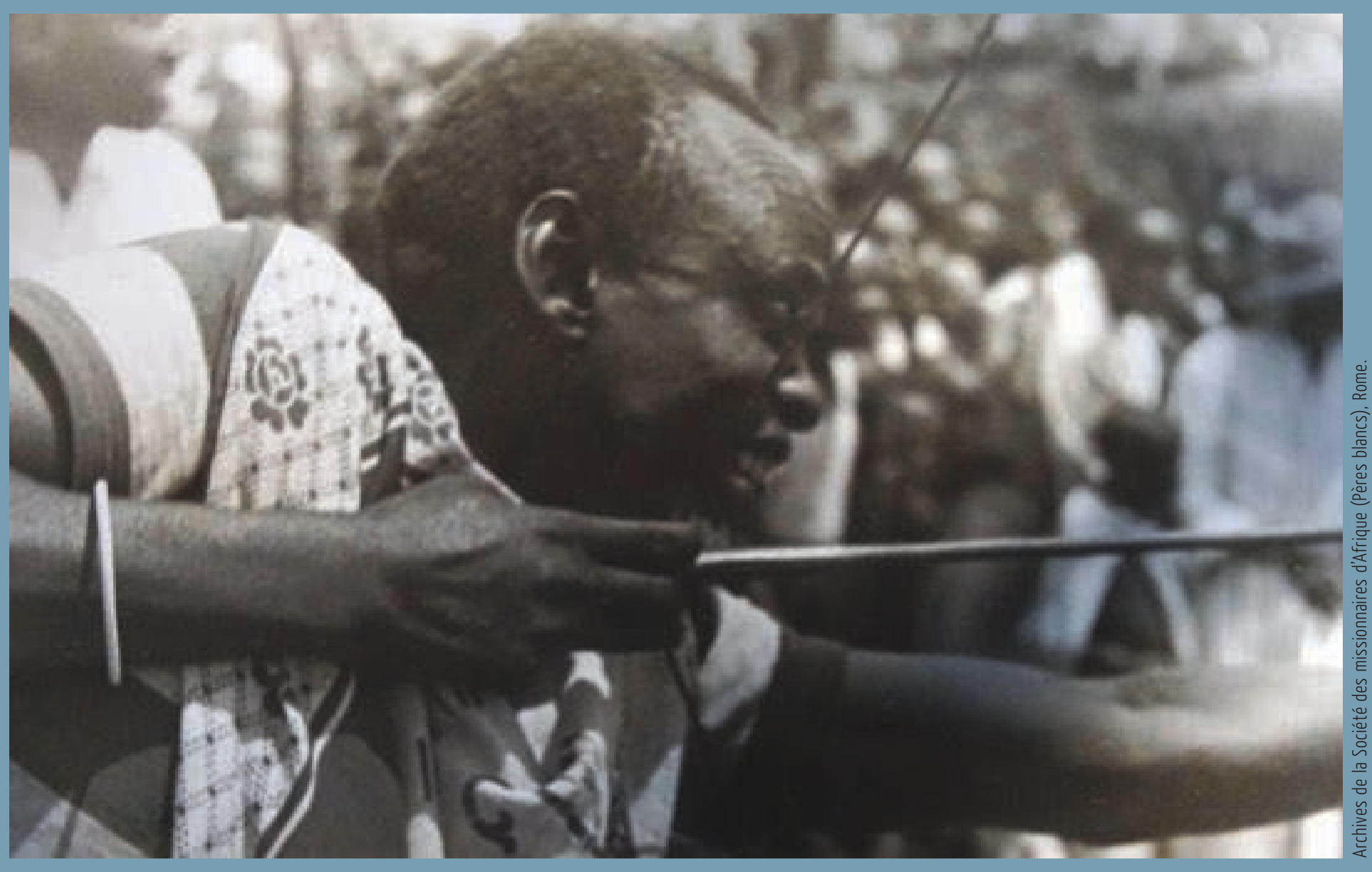




\section{DU COMBAT}

\section{Jouer et faire la guerre en Afrique (post-)coloniale et contemporaine}

"Quelques coups de sonde lancés dans différentes civilisations et périodes mettront en lumière l'élément agonal, c'est-à-dire ludique de la guerre."

Johan Huizinga, 1951

Ces trente-cinq dernières années, de nombreux États africains confrontés à de nouvelles tensions sociales, économiques et politiques y ont répondu par la guerre, de l'ordre des campagnes civiles à celui du monde milicien (Clapham 1998), de la milicianisation des institutions de combat (Debos 2013) au massacre d'État (Dumas 2014; Taylor 2000). Un grand nombre de publications scientifiques ont analysé les conditions de leur gestation, tout autant que les mobilisations individuelles et collectives affectées à leur exécution. Mais de fait, ces analyses se concentrent bien plus sur la guerre dans ses dimensions et implications politiques, religieuses ou économiques que sur les conduites concrètes de ceux qui la font. En Afrique comme ailleurs, «l'anthropologie du combat reste à faire», soulignait Jean-Pierre Warnier (2009) dans une lecture de l'ouvrage «pionnier» Combattre de Stéphane Audoin-Rouzeau (2013).

Sous un angle anthropologique, l'action de combattre s'apparente à l'ensemble des conduites sensorielles, affectives et motrices engagées dans les techniques de lutte du combattant ${ }^{1}$. Notre ambition est d'approcher la culture matérielle du combat en Afrique (post-)coloniale et contemporaine, autrement dit les conduites déployées dans le cadre de la subjectivation politique du combattant. Pour les comprendre, nous proposons d'engager un travail généalogique explorant des modes de formation non directement militaires, qui contribuent à l'intériorisation d'un ensemble de techniques et de dispositions susceptibles d'être réinvesties, plus tard, dans le combat. En d'autres termes, il nous semble que la 
question de la construction du combattant avant que celui-ci n'entre très concrètement en action est un préalable indispensable à l'appréhension de ses conduites martiales.

Parmi les nombreuses technologies explorées dans l'analyse des conflits qui ont touché la Sierra-Leone (Hoffman 2007), l'Angola (Weigert 2011), la Côte d'Ivoire (Marshall-Fratani et Richard Banegas 2003) ou encore la République démocratique du Congo (Prunier 2009) (armement, communications, flux financiers...), la matière première de la guerre - à savoir le combattant - apparaît en filigrane. Il faut dire que cette approche de la culture matérielle du combat - et donc de la subjectivation politique du «faiseur de guerre» (le sujet-combattant) - est soumise à une entreprise complexe de déconstruction des techniques et imaginaires engagés dans la mobilisation des combattants. Si la guerre ne peut se faire sans sujets, ceux qui la mènent ne peuvent la faire sans la matière de leur progressive sujétion à l'action de combattre. Le sujet-combattant est le produit de cet agencement. Notre ambition est d'explorer quelques modalités ludo-motrices affectées à sa subjectivation, prises comme des dispositions praxiques potentiellement mobilisables dans l'expérience «sensori-affectivo-motrice» du combat ${ }^{2}$. Depuis des décennies, il semble qu'elles assurent une intermédiation très efficace entre les processus quotidiens de formation des communautés africaines et les actions redoutables de groupes de militaires, miliciens ou civils déterminés à éliminer leurs opposants réels et/ou imaginés (Deleuze 1990).

\section{Du combat ordinaire...}

\section{Lutteurs ougandais, période coloniale}

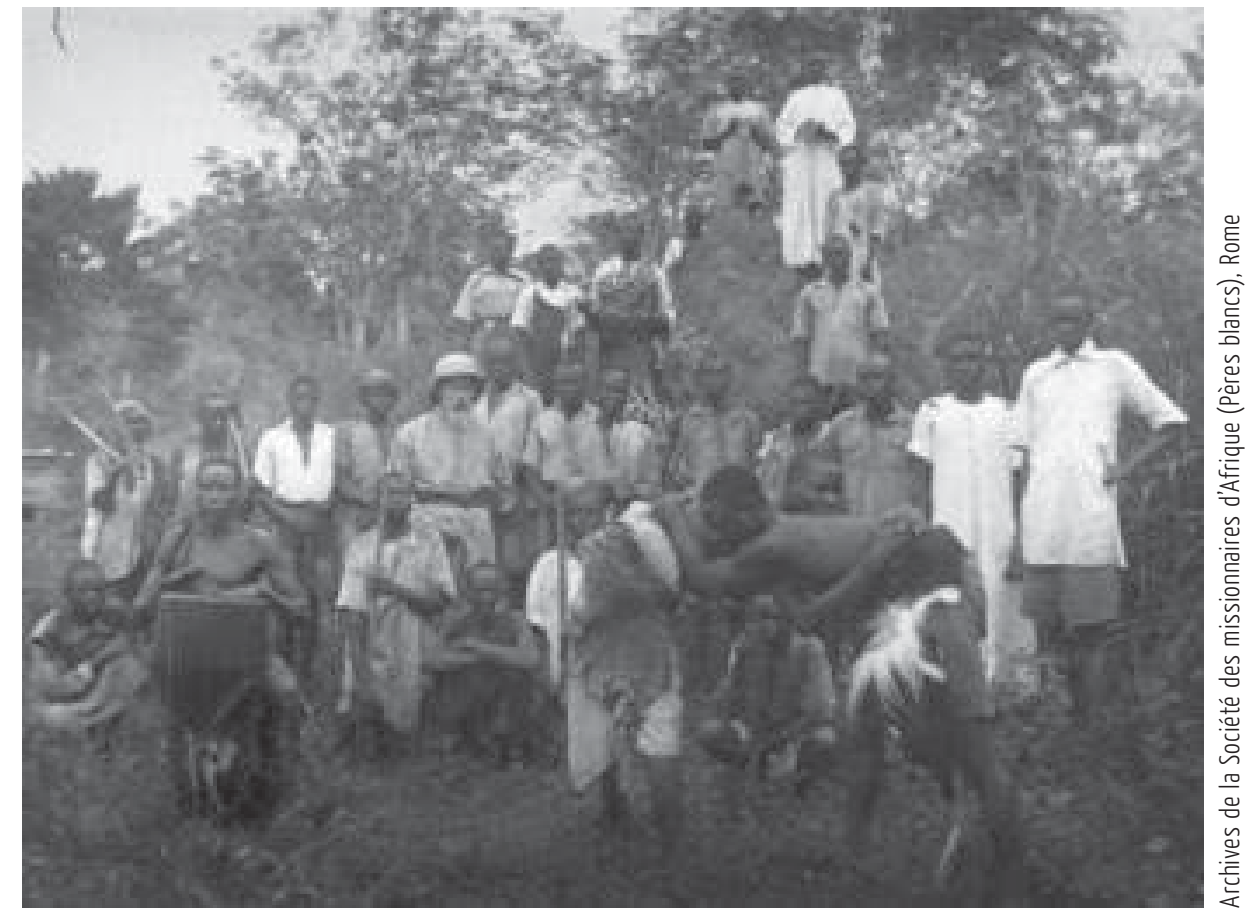


En Afrique comme ailleurs dans le monde, toute action ludo-motrice du quotidien ne mue pas en technique de combat. Les circonstances - sociales, économiques et politiques - sont en effet déterminantes. Avec elles, nous nous posons la question suivante: en quoi les jeux les plus quotidiens de certaines communautés africaines, les jeux de semailles et leurs déclinaisons locales, les parties individuelles (Chevé 2014) et d'équipe (Riot 2010), les danses (Ranger 1975) et leurs disjonctions guerrières (Nkulikiyinka 2002; Riot 2011), peuvent être considérés comme des techniques susceptibles de se trouver réinvesties dans le combat le plus concret?

Dans la mesure où ces communautés entrent en guerre (ce qui n'est bien évidemment pas toujours le cas), il s'agit en premier lieu de procéder à une brève observation des conduites sensori-motrices mobilisées et des «armes» qu'elles utilisent: tir, chasses, sorcellerie, éventrements, mises à feu, courses... Le répertoire est proportionnel au champ des possibles de l'action de combattre et à celles qui consistent à terroriser et/ou à anéantir son ennemi. De là, il faut reconnaître que l'instruction militaire ou milicienne (souvent brève et partielle) n'est peut-être pas le lieu central de la construction du combattant, et que - pour le dire simplement - il y a une vie avant le combat.

Dans une longue notice de condoléances portées à l'un de ses anciens camarades de jeu, un ancien scout catholique révèle qu'entre 1957 et 1959, le Père Joseph De Laet (plus connu sous le nom de Père Buffalo) organisait pour une section d'enfants de la mission Saint Paul de Burumbu (une succursale de Léopoldville, actuelle Kinshasa) des jeux de pistes urbains destinés à « rattraper» la jeunesse « délaissée» des environs pour l'intégrer aux activités de la jeunesse catholique du centre (le mouvement xaveri) : «Nous voulons la jeunesse de la ville pour le Christ», telle était la devise de la section ${ }^{3}$.

Dans la situation urbaine qui était la leur, ces adeptes des jeux de piste et de capture partaient à la conquête des traces laissées par la jeunesse non convertie des environs. Il

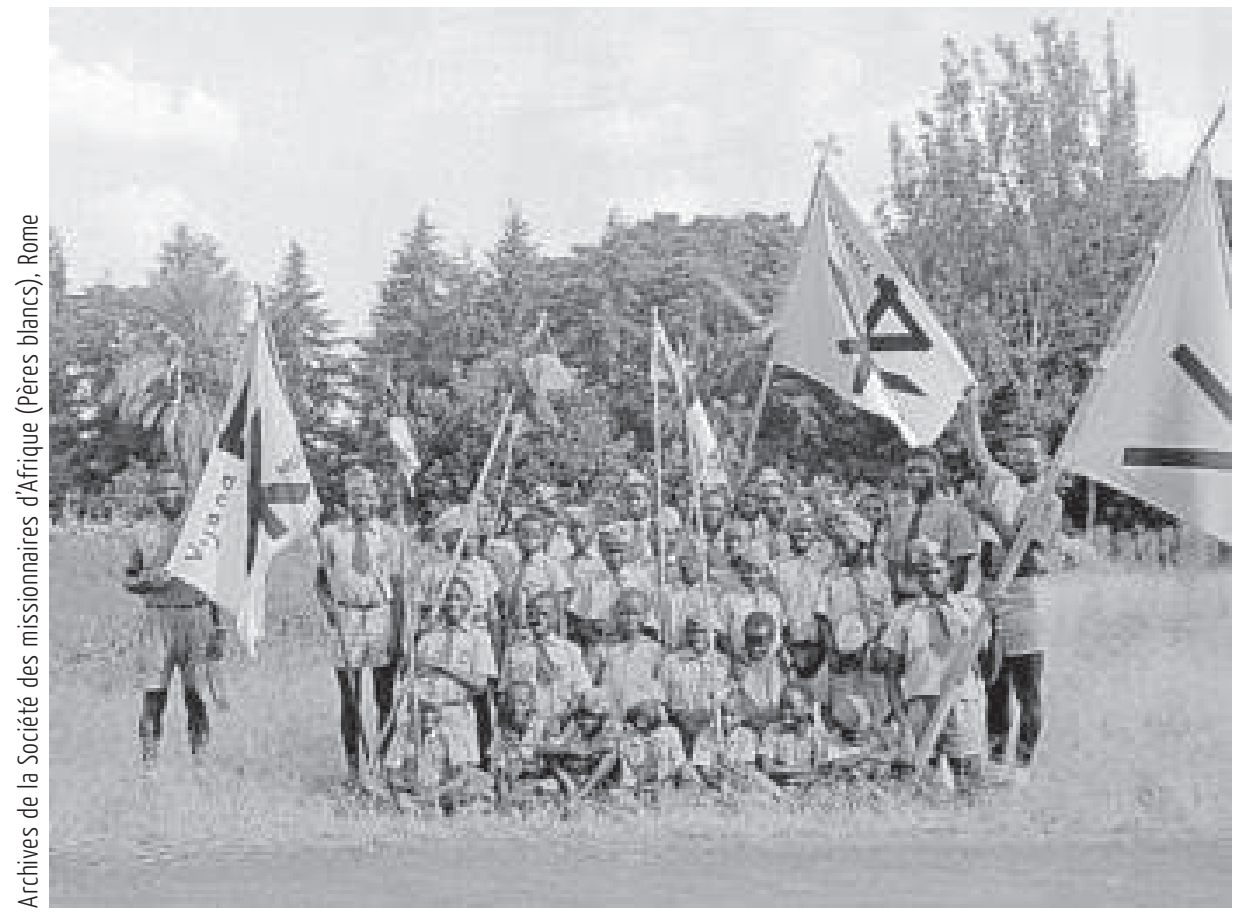

Une troupe de scouts catholiques en Afrique centrale 


\section{Jeunes milices en activité dans la région \\ de Kinshasa, République démocratique du Congo, années 2000}

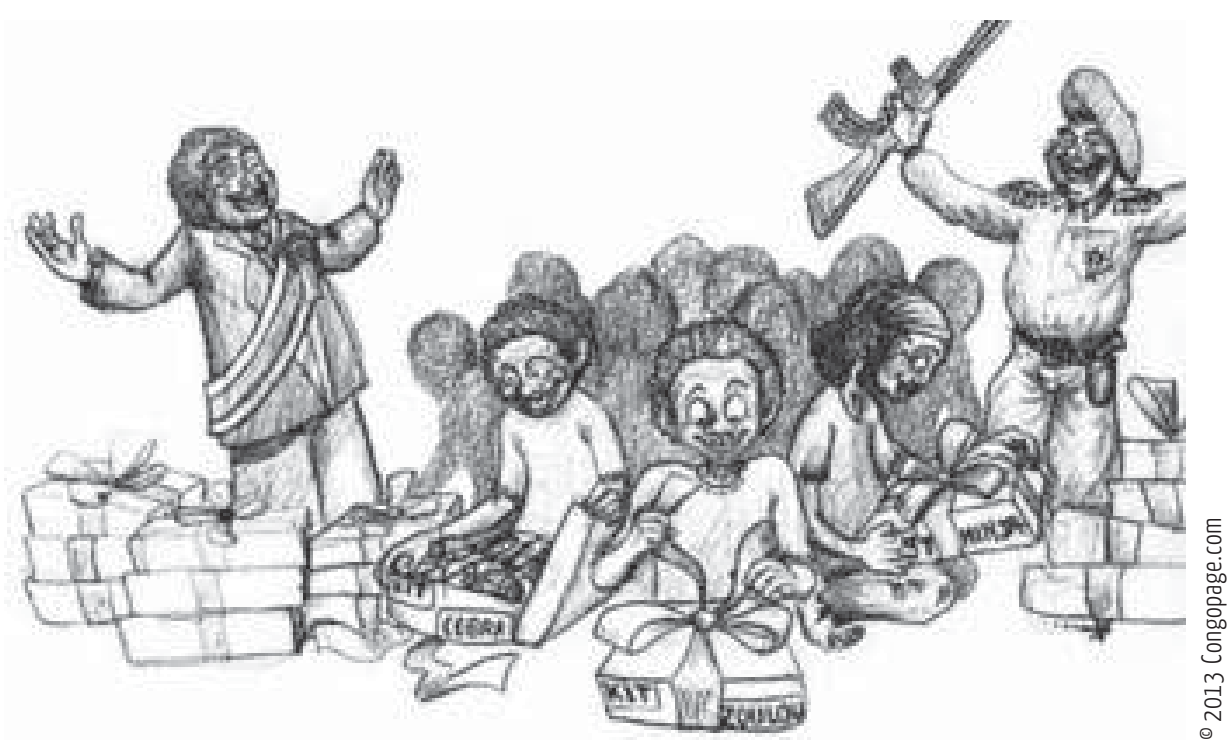

était bien question de jouer, dans la mesure où leurs actions pouvaient mener à la victoire de leur camp, à la condition près qu'ils parviennent à rattraper l'autre camp (par exemple un groupe de jeunes délinquants) avant son arrivée à «destination» (par exemple un lieu de consommation de boissons alcoolisées, ou la prison).

Ainsi formulées, ces actions faisaient appel à de la sémiotique (lecture et signification des signes qui constituent la piste), de la feinte (fausses pistes), de la conquête de territoire (balisage et appropriation du terrain), et de la capture (de traces matérielles selon un objectif de conquête de l'espace du camp adverse). Les éléments mobilisés par les joueurs étaient bien évidemment proportionnels au champ des possibles de leurs conduites. Par de multiples contraintes, ruses, coups portés, renversement des rôles, ils intériorisaient les actions qui les construisaient comme sujets de leur propre jeu; et sous l'effet de règles dictées depuis la transposition civile d'un dispositif missionnaire, ils s'identifiaient - par les mêmes actions - au jeu de leurs rivaux ${ }^{4}$.

Assemblées en un mouvement connu sous le nom de bilisme, les dimensions de capture et de frontière de ces conduites ludiques urbaines s'inscrivirent dans un univers polymorphe où furent entremêlés le monde du chasseur précolonial, la scène prédatrice des diamants de la région et la fabrication locale des imaginaires du Far West. La jeune figure du «cowboy chasseur» naquit à la toute fin des années 1950, et se renforça immédiatement autour de leaders formés à l'exercice de puissantes compétitions entre gangs rivaux. À partir de novembre 1960, la scène des émeutes de Léopoldville se nourrit de l'apparition d'un nombre incalculable de nouveaux gangs qui, en référence aux Casques bleus de l'ONU, passèrent immédiatement de la figure de «l'écumeur du Far West » à celle du soldat (De Boeck 2004).

Au tournant de l'indépendance de l'ancien Congo belge, la situation de ces jeunes scouts convertis (en trois années) en combattants urbains nous ramène à la question du passage de l'ordinaire à l'extraordinaire, c'est-à-dire à la mutation potentielle de conduites quotidiennes de jeu en armes du combat concret. Aussi, comment se fait-il que sans instruction militaire (ou administrée en quelques semaines), un homme « ordinaire » se montre capable de mener les actions les plus meurtrières ${ }^{5}$ ? 
Par exemple, comment pourrait-on dire que dans le courant des années 1980, un agriculteur rwandais qui pratiquait le jeu de semailles igisoro se préparait à utiliser un outil agricole pour assassiner - en avril 1994 - l'un de ses voisins? Il ne s'y préparait pas. Face à son adversaire-compagnon de jeu, il jouait à planter et à récolter.

L'igisoro est constitué d'une plaque de bois rectangulaire, creusée de quatre rangées de huit cavités. Chaque joueur commence par recevoir trente-deux graines (les «pions »). Le but de la partie est de prendre toutes les graines de l'adversaire. À l'entrée d'une habitation, les joueurs se placent de part et d'autre de la plaque de jeu. Le camp de chaque joueur est constitué par les deux rangées qui se trouvent de son côté. On joue dans le sens contraire aux aiguilles d'une montre. Les cavités des deux rangées centrales reçoivent quatre graines chacune. Le premier joueur est tiré au sort. Les parties suivantes sont commencées par le vainqueur de la partie précédente. À chaque tour, le joueur prend toutes les graines d'une cavité au choix de sa zone de jeu; il les sème ensuite une à une dans les cases qui suivent la cavité d'origine du mouvement. On ne sème pas chez l'adversaire mais dans sa propre zone de jeu.

Ainsi fait, le jeu d'igisoro consiste à mobiliser un ensemble de coups et de ruses destinés à prendre le maximum de graines à son adversaire, tout en l'empêchant de semer les siennes. Au Rwanda comme ailleurs, les circonstances ont été déterminantes. Au même titre que la houe était le principal emblème de subjectivation des agriculteurs hutu, l'action de semer était leur technique la plus quotidienne. Elle rejoignait la matérialité d'une identification plus large au « défricheur». Certains de leurs voisins tutsi, plus ou moins éloignés dans l'espace, en avaient d'autres: costumes à l'occidentale, taille longiligne, maîtrise du français... Dans une situation propice à la prédation économique, à la racialisation du politique - notamment par le biais du

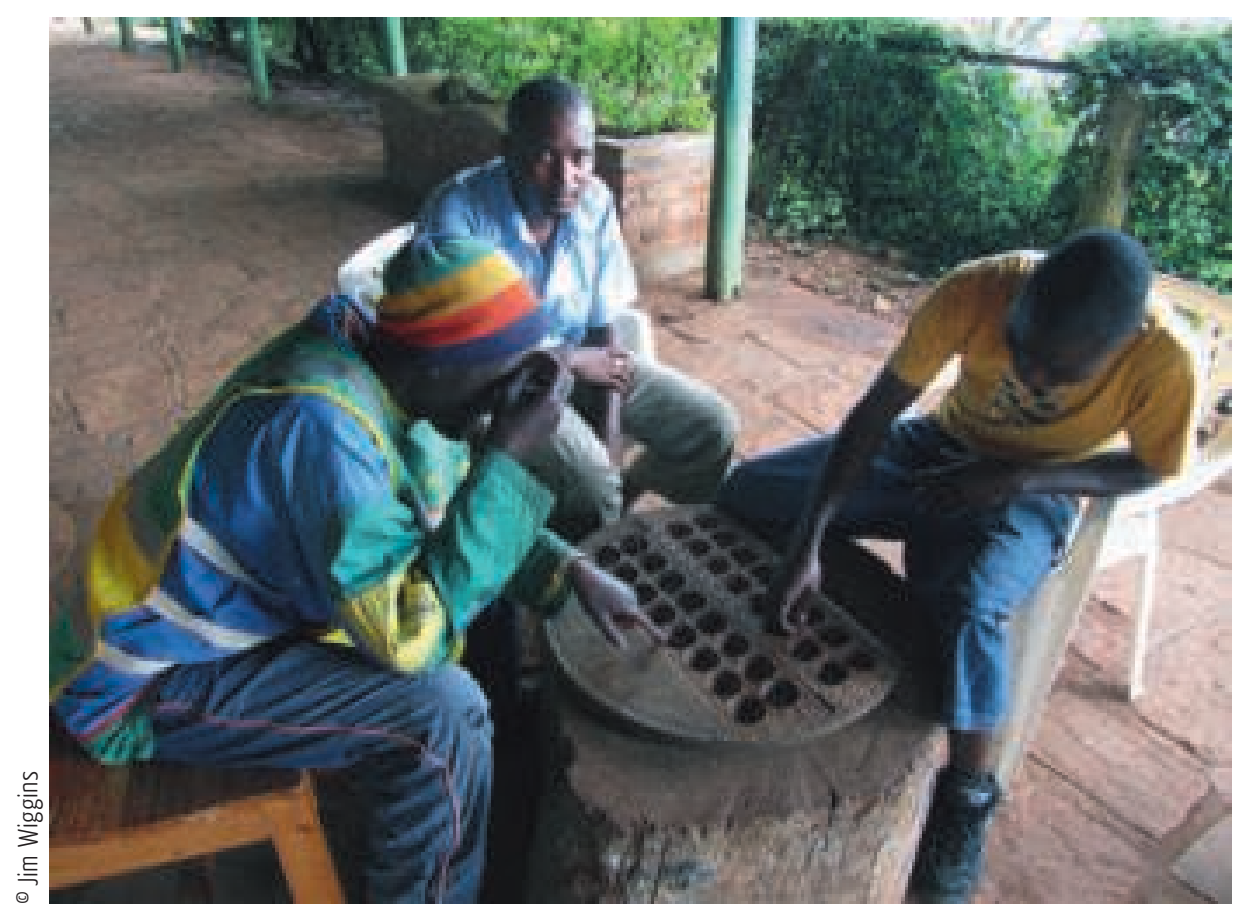

Joueurs d'igisoro 
mouvement extrémiste Hutu power - et au développement d'un sentiment d'insécurité - autour du conflit qui opposait depuis le début des années 1960 les forces de la « République hutu » à celles du front peu à peu constitué en Ouganda par des réfugiés Tutsi du Rwanda -, des agriculteurs (en réalité des dizaines de milliers) ont identifié ces derniers emblèmes à ceux d'un «ogre tutsi ».

Il semble qu'au fil des années, les jeux de «pillage» de cet «ogre», soit l'appropriation des ressources culturelles et économiques les plus convoitées, aient alimenté le sentiment d'humiliation et de frustration du «semeur». Dans la situation que nous évoquons, l'action ludique et disjonctive qui consistait à planter-récolter, soit à semer et à «prendre à l'autre» par la mise en mouvement de tout un répertoire de ruses et de coups, pourrait avoir créé une mémoire sensori-motrice susceptible de cheminer depuis le «champ » du jeu vers sa reconversion meurtrière. Une part du glissement ainsi constaté s'oriente vers une technologie culturelle de la terre productrice d'un « défricheur hutu » peu à peu radicalement opposé au style de vie des «évolués tutsi » - «l'ogre»- des environs (De Lame 1996; Vidal 1991).

Nous nous éloignons donc de l'idée utilitariste qui associerait la « fonction » meurtrière de l'objet à son utilisation initiale. Car le sujet-combattant n'est pas simplement assujetti à des objets, des savoirs et des techniques potentiellement mobilisables dans le champ matériel de la violence physique. Qu'il soit contraint ou volontairement engagé dans l'action meurtrière, il y emmène des ressources incorporées depuis son plus jeune âge et les mobilise dans l'ensemble des actions affectées à l'atteinte ou l'élimination de sa cible. Ceci n'est pas un choix, mais un processus inconscient de manifestation de conduites sensori-affectivo-motrices précédemment acquises, et initialement étrangères au champ matériel des actes les plus redoutables dans lesquels elles seront à nouveau mobilisées. Il n'est pas nécessaire de transformer le manche de la houe en lance pour en faire une arme. Ce qui compte le plus est l'expérience de celui qui le manie. La question n'est donc pas de savoir ce qui, à un moment donné, pousse un homme à utiliser une houe comme une arme d'attaque ${ }^{6}$. Elle nous invite plutôt à explorer le régime ainsi créé, qui se rapporte à la transposition agonistique d'une technique précédemment acquise dans un univers ludique, et ajustée à une nouvelle situation. Ce régime nous semble constituer un élément important d'une anthropologie praxéo-politique plus large du combat - c'est-à-dire l'étude de la subjectivation politique des conduites mobilisées avant, pendant et après l'action de combattre. 


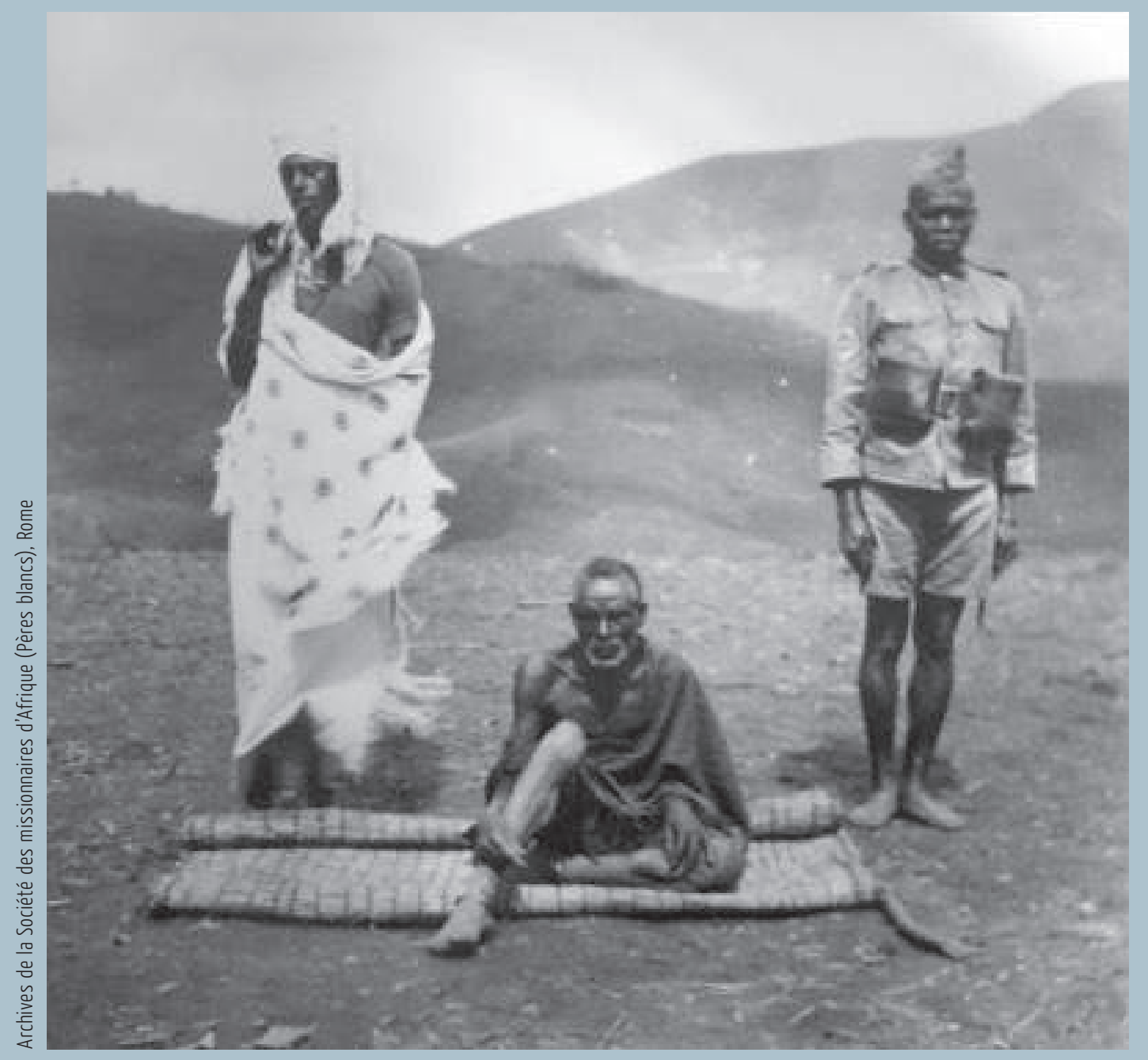

Temps colonial, un agent de la force publique et un chef tutsitiennent prisonnier un agriculteur assimilé à l'ethnie hutu 


\section{... à la ludo-motricité du combattant}

Parmi les actions ludiques, nous avons vu que certaines, comme les jeux de semailles, ne font pas directement référence au combat, ou imagine et matérialise celui-ci sans aller jusqu'à l'élimination physique de l'adversaire, réel ou plus ou moins imaginé. D'autres, plus proches d'un univers diairétique (coupant, tranchant), sont apparentées à ce que nous pourrions appeler une «ludo-motricité agonistique».

Dans plusieurs pays africains, le glissement politique pris par un certain nombre de jeux a parfois alimenté une violence extrême. Nous avons observé que dans l'ancien Congo belge, des parties de «capture» ont alimenté la reconversion milicienne de groupes de jeunes urbains convertis en nouveaux prédateurs des ressources minières de la région. L'initiation ludo-motrice des personnes qui composent ces groupes est un impensé de leur genèse et de leur mobilisation. Les conduites sensori-motrices productrices de leur sujétion au combat le plus concret sont parfois citées, mais rarement étudiées. Il est pourtant possible de les aborder sous l'angle des transferts de savoirs ludo-moteurs vers des situations de combats, de guerre et de massacres, mais aussi vers l'appareil technologique plus large du combat mené par des gouvernements de type militaire, civil ou milicien.

Danse guerrière au Rwanda

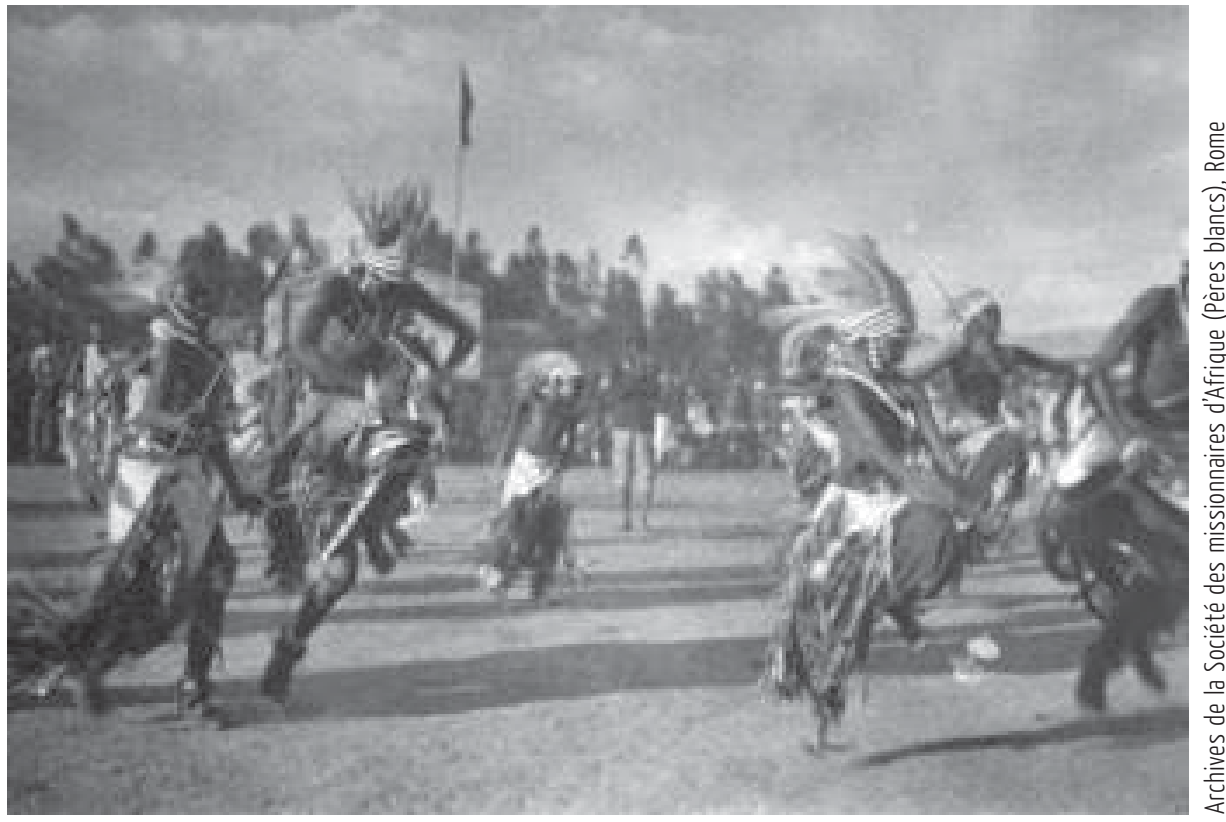

Affectée à un enjeu politique plus large - allant jusqu'à l'élimination systématique des opposants -, la situation ainsi créée renvoie sans aucun doute à la «double action » (Bayart 1994) de l'instrumentalisation politique des mouvements et de l'adhésion à des répertoires culturels qui participent à la formation des communautés politiques africaines. Pourtant - et bien que l'instrumentalisation puisse s'envisager par-le-bas ${ }^{7}$ - le transfert du combat ludique à la ludo-motricité du combattant est plus proche de l'action directe 
et de la possibilité (sous l'effet de la mémoire sensori-motrice et selon des circonstances déterminantes) de la réajuster en technique martiale. Outre le fait qu'une telle approche puisse lever l'un des voiles de la subjectivation des ressources agonistiques de l'État (si diffracté qu'il soit) en Afrique (Bayart 1993), le répertoire ludique des ruses et des coups s'en trouve assujetti à son éventuel transfert dans l'univers du combat concret.

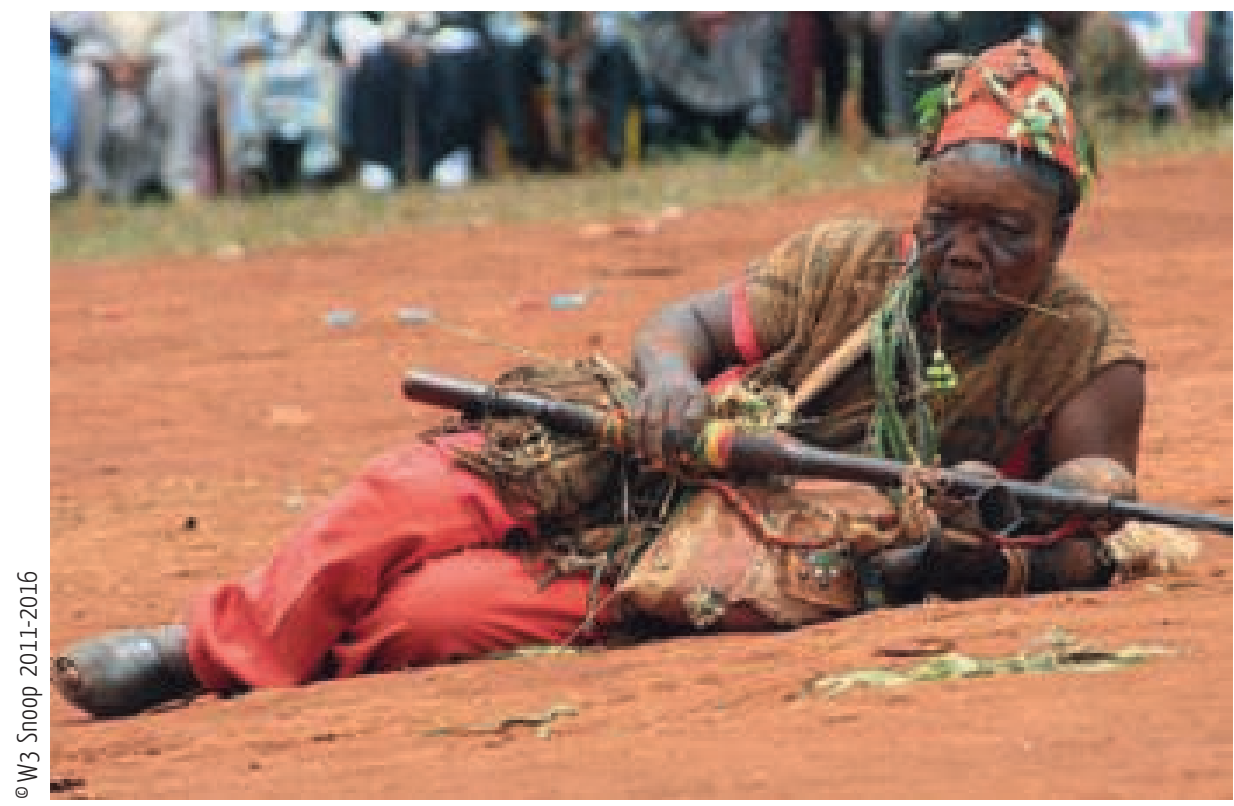

Sur le terrain des pratiques, le corpus des pratiques culturelles africaines communément associées au «corps» et au «loisir» (danses, activités sportives d'origine occidentale, jeux guerriers) a été régulièrement associé aux manifestations techniques et matérielles du politique. Les technologies culturelles et guerrières, étudiées par Jean-Pierre Warnier à l'aune de la construction des sujets Mankon du roi Ngwa'fo au Cameroun, ont été l'un des révélateurs du phénomène.

«Quel est l'effet de ces actions sur les sujets? [...] c'est une intériorisation qui permet de construire le sujet en tant que sujet de ses propres actions guerrières, et de ses propres conduites sensori-affectivo-motrices en situation de combat plus ou moins imaginaire » (Warnier 2002).

Si la mesure de cet «effet » est indiscutablement complexe, il peut être analysé sous la matière et les conduites affectées à des jeux définis par des règles, alimentés par des ruses converties en coups. Parmi les règles se trouvent, par exemple, celles de jeux de capture ou de conquête d'un territoire. Parmi les ruses et la mètis (Detienne \& Vernant 1974) qui peuvent s'y inscrire se trouvent la feinte, la prévision, ou encore la saisie d'opportunités. Parmi les coups se dégagent certains directement portés sur l'adversaire ou ceux, plus insidieux, qui portent atteinte à son affectivité. Ensemble, règles, ruses et coups s'inscrivent dans la mémoire sensori-motrice du joueur, constituant la matrice intériorisée et sans cesse réactualisée des techniques ludiques et/ou martiales mises en œuvre depuis l'enfance.
La guerre mise en scène au Cameroun 
En Ouganda, l'ancien chef d'État Idi Amin «Dada» était dôté d'une mémoire ludomotrice. Une composante peu explorée de sa biographie révèle que ce dernier, avant d'organiser le coup d'État militaire qui le porta au pouvoir en 1971, et avant de faire de la destruction de la vie l'une de ses politiques les plus quotidiennes, avait intégré l'armée coloniale britannique comme commis de cuisine ${ }^{8}$ (en 1946), puis fait de la boxe son art majeur (entre 1951 et 1960). Sous l'étude comparée du «soldat Amin» et du «boxeur Ali », Ali A. Mazrui a montré le rôle joué par la «face sportive» d'Idi Amin «Dada» dans sa conquête du pouvoir. Au-delà de l'aspect biographique, l'analyse de l'auteur ouvre des pistes passionnantes quant à la question de comprendre comment se sont formées les acquisitions ludo-motrices du personnage, et d'expérimenter la possibilité de remonter à ces dernières, à partir de ses pratiques une fois le pouvoir conquis.

«Amin [...] had a brutal side to his personality. Even before he attained supreme political power in Uganda, he had known moments of brutal urges and inclinations. He had killed many as a soldier, just as he had defeated as a boxer» (Mazrui 1977).

Idi Amin, L'ogre de Kampala

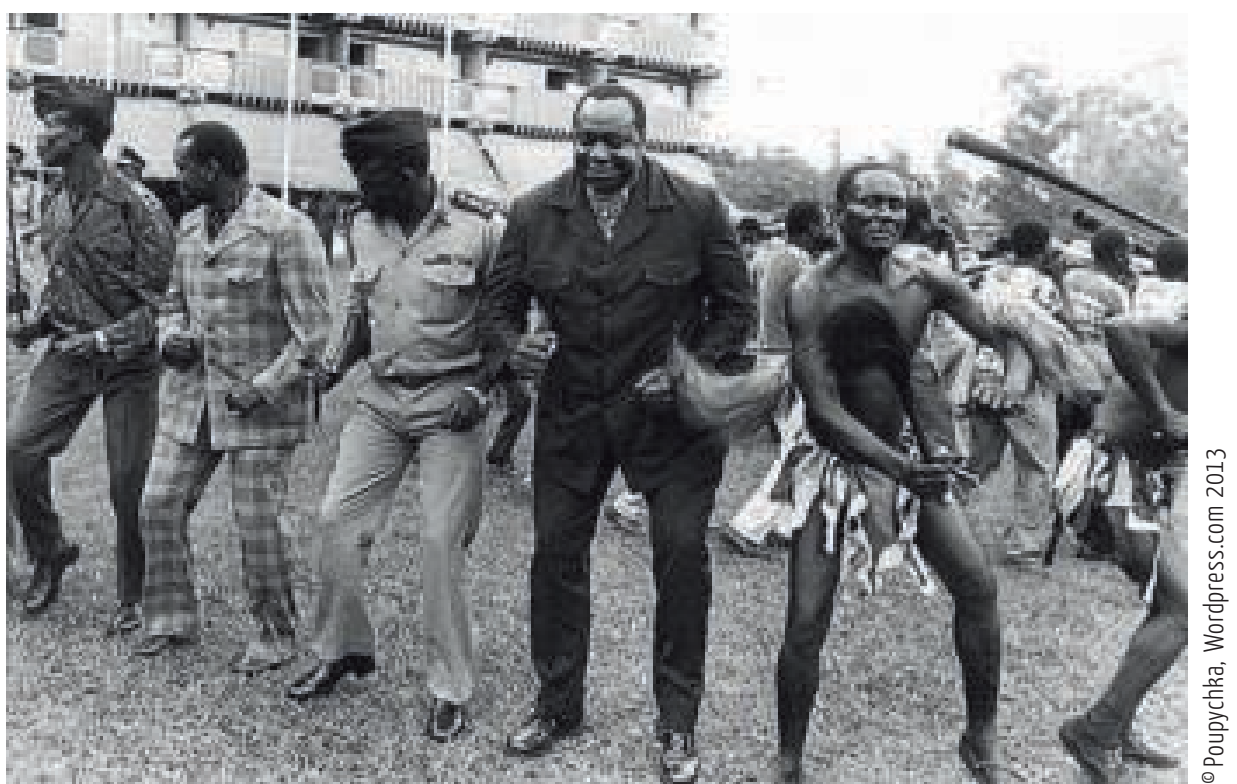

\section{Mémoire et concurrence}

De très périlleuses observations de terrain pourraient nous aider à préciser cet «effet de mémoire $»^{9}$. Une autre possibilité nous est offerte, en cela que les récits meurtriers nous aident à préciser la phénoménologie des techniques utilisées. Quand un ancien groupe de génocidaires narre à Jean Hatzfeld leur Saison de machette (Hatzfeld 2003), le journaliste recense en premier lieu le geste de leur action, conduite dans le répertoire plus large d'un métier: le «travail de coupe» des Tutsi du Rwanda. Les opérations disjonctives de la danse guerrière ont-elles « aidé » certains d'entre-eux à accomplir leurs actes? Peut-être, si l'on pense aux chasses menées par d'anciens danseurs dont la tâche (qui ne consistait plus à danser) avait été transformée en traque des Tutsi du pays; et peut-être encore plus 
si l'on observe le fait que ces groupes de danseurs ont constitué un vivier important du recrutement d'un grand nombre de miliciens interahamwe (Riot 2014).

Dans le cadre de la participation de certains jeux à l'accomplissement du génocide des Tutsi au Rwanda, comme dans celui de la biographie pugilistique d'Idi Amin «Dada » ou de celle - plus collective - des soldats-prédateurs de la République démocratique du Congo (RDC), nos préoccupations résident bien plus dans la généalogie ludo-motrice des mobilisations agonistiques que dans une insasisfaisante opération déductive du passage du jeu au combat. Ainsi formulées, nous proposerions de les situer sous deux modalités.

D'une part, sous l'aspect de la mémoire du sujet-combattant, qui donne toute son importance à la biographie et aux trajectoires des membres d'un « corps d'attaque». Après avoir acté leur mobilisation dans le combat concret, on peut ainsi convenir de dégager la part ludo-agonistique de leurs acquisitions plus anciennes et moins documentées. D'autre part, sous l'aspect ludo-compétitif de corporations combattantes susceptibles d'ajuster leurs coups à la mesure de leurs partenaires de guerre convertis en émulateurs des actes les moins ludiques qui soient. Sous ces deux modalités, nous assisterions très probablement à la mise en forme ludique de techniques affectées à une part de la construction du combattant. Il convient ainsi de poursuivre l'exploration du sujet-agonistique, susceptible de réagir selon les relations de réciprocité qui gouvernent sa subjectivation à des actions des plus ordinaires aux plus meurtrières.

Comme le formule Michel de Certeau, cette mémoire (qui est le produit de «l'effet» dégagé par Jean-Pierre Warnier) « [...] suppute et prévoit les voies multiples de l'avenir en combinant les particularités antécédentes ou possibles» (De Certeau 1990). De ce point de vue, le «tour» qui pourrait caractériser le glissement du jeu vers le combat « implique d'abord la médiation d'un savoir, mais un savoir qui a pour forme la durée de son acquisition et la collection interminable de ses connaissances particulières» (De Certeau 1990).

Les jeux de notre objet font en effet appel à d'interminables connexions. Si le chercheur ne peut jamais s'enorgueillir d'avoir atteint la «lune» des conduites, il convient

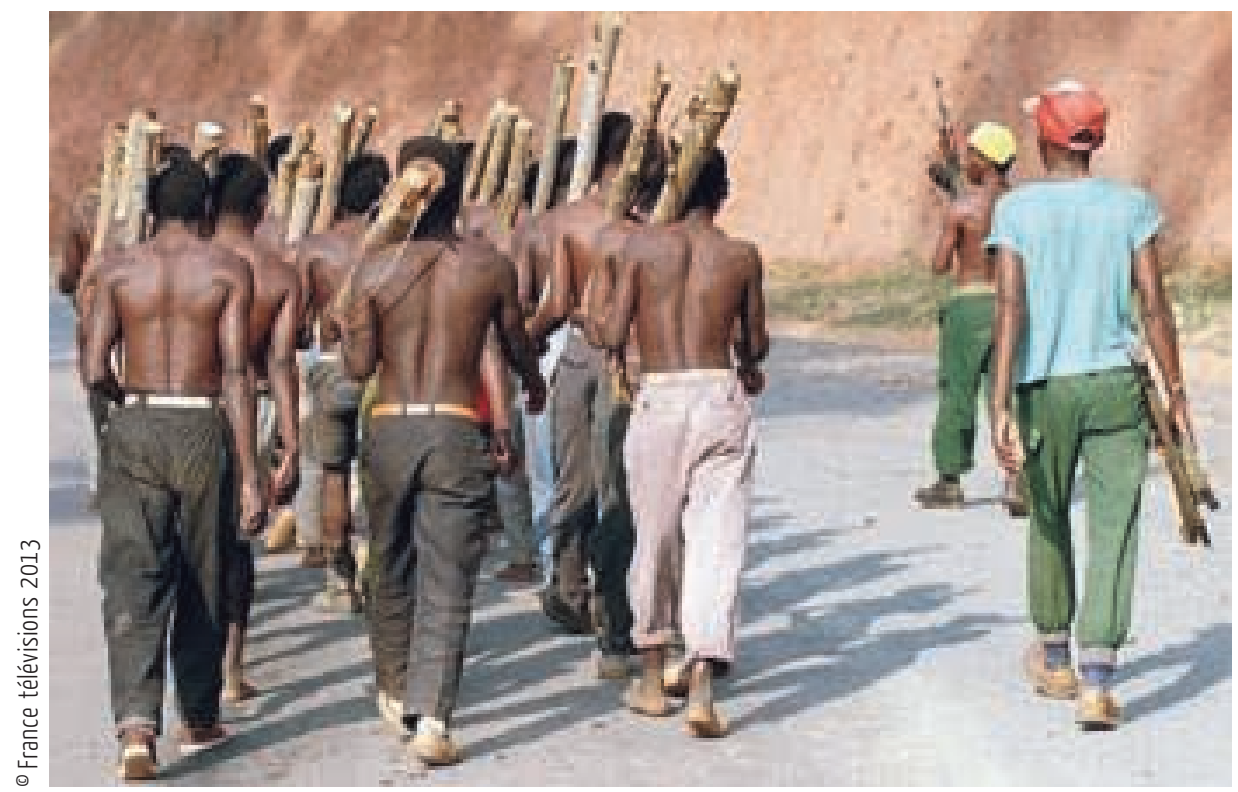

Milice extrémiste au Rwanda 
de caractériser la formation d'un savoir susceptible d'établir les liens entre le combat ludique et la mise en jeu des actes les plus redoutables. Une hypothèse nous dirige provisoirement vers le «savoir-pouvoir » d'une lutte compétitive susceptible de conduire la subjectivation politique de combattants dotés de la mémoire des règles, des coups et des ruses acquis dans l'infini de leurs opérations disjonctives.

Dans une réflexion sur l'impérialisme, Paul Veyne nous invite à interroger le polymorphisme de l'agôn dans les distinctions nécessaires à son objectivation dans le monde de la « guerre ludique» et celui des «professionnels de la guerre», précisant que « [...] s'il y a compétition, à la guerre, c'est entre combattants d'une même armée» (Veyne 1975).

La distinction entre le combat ludique et le combat concret ne fait aucun doute. Ce que nous explorons est le glissement potentiel de l'un vers l'autre, ceci sous les trajectoires ludo-motrices «ordinaires» des «professionnels de la guerre». Il s'agit de l'une des composantes de leur biographie, grâce à laquelle nous pouvons supposer que «la compétition entre combattants d'une même armée » constitue l'un des marqueurs du transfert constaté - dans lequel défis, concurrence entre combattants et autres récits de leurs exploits avant, pendant et après le combat ajouteraient de l'émulation compétitive à leur action et renforceraient, in fine, l'efficacité des actes les plus redoutables. Sous la construction d'un «agôn-mutant», ce «phénomène» est celui de la subjectivation ludomotrice du combattant amené (ou pas) à devenir membre d'une corporation de combat.

\section{Disjonction}

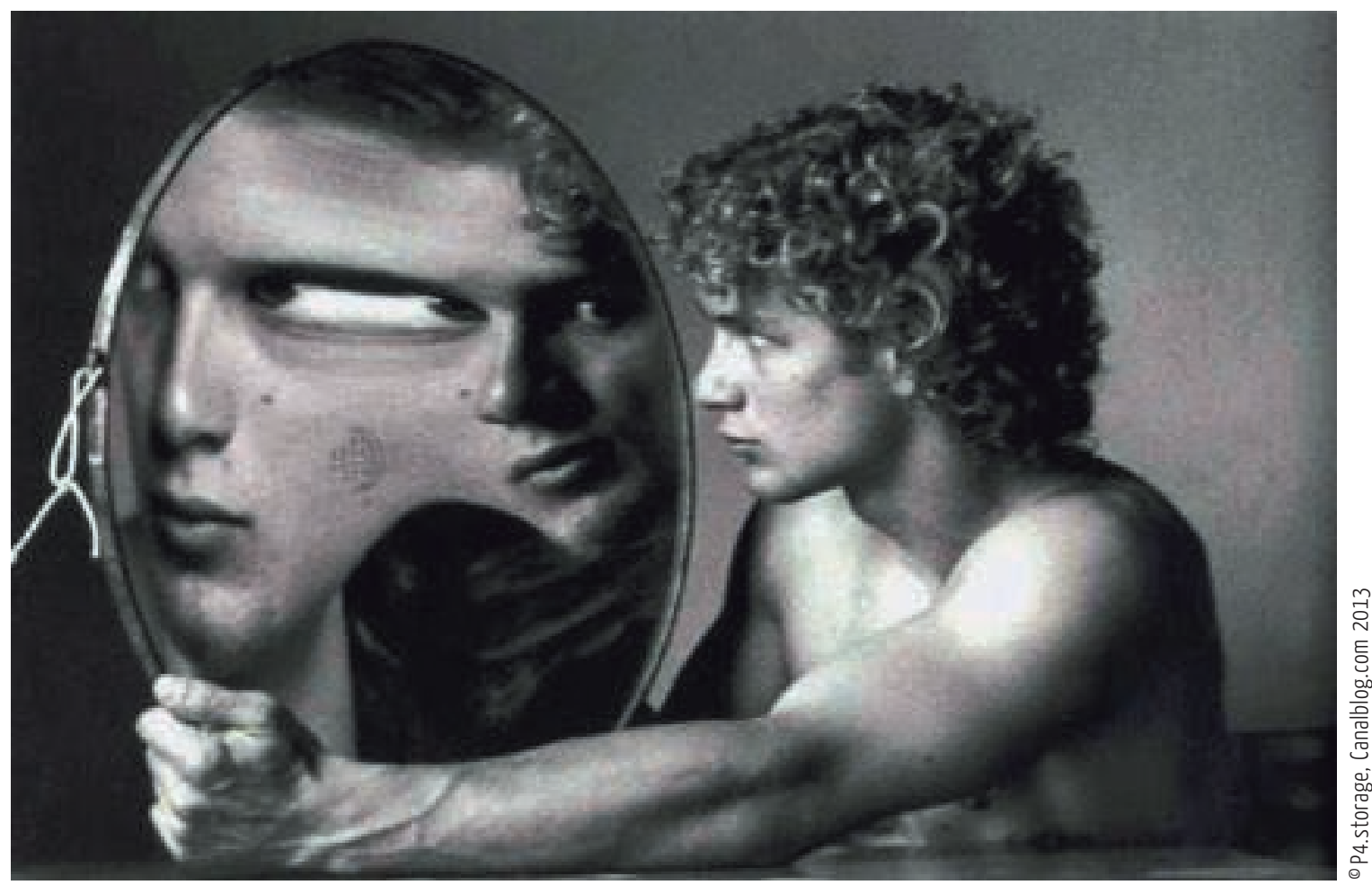




\section{NOTES}

Photo d'ouverture: Tireur à l'arc, Rwanda. Archives de la Société des missionnaires d'Afrique (Pères blancs), Rome.

1. Ainsi se différencient le guerrier et le combattant, tout comme les approches historiques et anthropologiques de la guerre et du combat (Warnier 2011).

2. Nous rejoignons en ces termes l'anthropologie praxéologique du politique, des travaux issus de l'association du socio-politiste et de l'ethnologue (Bayart, J.-F. \& J.-P. Warnier 2004).

3. Nous avons ici recoupé plusieurs informations contenues dans les deux pages Internet suivantes: www. facebook.com/gode.bayama/posts/641667585843758 et www.digitalcongo.net/article/61834, que nous avons croisées aux propos de Philippe De Boeck (2004).

4. Le jeu est ici une forme locale et urbaine des jeux de piste présentés par le Père Defour, fondateur du mouvement xaveri dans l'ancien Congo belge. Nous avons consulté les premières brochures du mouvement aux archives de la Société des missionnaires d'Afrique (Pères blancs), Via Aurelia, Rome.

5. Souvent très sommaire, l'instruction militaire (ou milicienne) n'est qu'un passage vers le combat concret. Il n'est pas nécessaire de revêtir l'apparat du combattant pour être et être considéré comme un «homme en armes». La question de «l'être combattant» n'est en effet pas la plus pertinente. Le plus important est sans doute de considérer les actions ordinaires engagées dans l'expérimentation sensori-affectivo-motrice de sa sujétion à des technologies postérieurement impliquées dans les actions très concrètes du combat le plus redoutable. Au Tchad, Marielle Debos propose de considérer l'hypothèse que «l'aboutissement de la carrière d'un homme en armes puisse précisément être de s'en passer » (2013).
6. C'est la question de «l'intention», qui est peu pertinente du point de vue d'une anthropologie praxéologique de la subjectivation politique, c'est-à-dire de l'étude des conduites concrètes affectées à la fabrication individuelle et collective des sujets du pouvoir.

7. Ceci dans la mesure où on la considère comme l'un des produits d'une identification plus large à des objets-instruments.

8. Comme tant d'autres pratiques de «l'ordinaire» et selon des circonstances déterminantes, les opérations d'(e) (al)chimie culturelle qui font la cuisine pourraient-elles rejoindre les glissades du jeu de toboggan qui caractérisent, dans le monde infini du transfert, la terrible descente du quotidien vers la violence physique? (De Certeau et al. 1994).

9. Grâce à son expérience d'observation (très) participante des combats menés par des guerriers Anuak aux confins soudano-éthiopiens, Edward EvansPritchard aurait pu y procéder. Le fait qu'il prit la tête de ce conflit en recrutant les guerriers par ses soins explique- t-il son silence? (Warnier 2009, 2011). 


\section{RÉFÉRENCES}

Audoin-Rouzeau, S. 2013 Combattre. Une anthropologie historique de la guerre moderne, XIX'-XXI siècle. Paris: Seuil.

Bayart, J.-F. dir. 1993 Religion et modernité politique en Afrique noire. Dieu pour tous et chacun pour soi, Paris: Karthala.

Bayart, J.-F. 1994 Lillusion identitaire. Paris: Fayard.

Bayart, J.-F \& J.-P. Warnier dir. 2004 Matière à politique. Paris: Karthala.

Clapham, C. dir. 1998 African Guerrillas. Londres: James Currey.

Chevé, D. et al. dir. 2014 Corps en lutte. L’art du combat au Sénégal, Paris: CNRS Éditions.

Debos, M. 2013 Le métier des armes au Tchad. Le gouvernement de l'entre-guerre. Paris: Karthala.

De Boeck, P. 2004 «La frontière diamantifère angolaise et son héros mutant », in J.-F. Bayart \& J.-P. Warnier dir. Matière à politique, Paris: Karthala: 93-128.

De Certeau, M. 1990 L'invention du quotidien, tome I: Art de faire. Paris: Gallimard.

De Certeau, M. et al. 1994 Linvention du quotidien, tome II : Habiter, cuisiner, Paris: Gallimard.

De Lame, D. 1996 Une colline entre mille ou le calme avant la tempête. Transformations et blocages du Rwanda rural. Tervuren: Musée royal de l'Afrique centrale.

Deleuze, G. 1990 Pourparlers, 1972-1990. Paris: Minuit.

Detienne, M. \& J.-P. Vernant 1974 Les ruses de l'intelligence. La mètis des Grecs. Paris: Flammarion.

Dumas, H. 2014 Le génocide au village, le massacre des Tutsi au Rwanda. Paris: Seuil.

Hatzfeld, J. 2003 Une saison de machette. Paris: Seuil.

Hoffman, D. 2007 «The Meaning of a Militia. Understanding the Civil Defence Forces of Sierra Leone», African Affairs: 639-662.

Huizinga, J. 1951 Homo ludens. Essai sur la fonction sociale du jeu. Paris: Gallimard.

Marshall-Fratani, R. \& R. Banégas dir. 2003 La Côte d'Ivoire en guerre: dynamiques du dedans et du dehors, Politique africaine 89.

Mazrui, A. A. 1977 « Muhammad Ali and Soldier Idi Amin as International Political Symbols: The Bioeconomics of Sport and War », Comparative Studies in Society and History 19: 189-215.

Nkulikiyinka, J.-B. 2002 Introduction à la danse rwandaise traditionnelle. Tervuren: Musée royal de l'Afrique centrale (Annale, vol. 166).

Prunier, G. 2009 From Genocide to Continental War. The «Congolese» Conflict and the Crisis of Contemporary Africa. Londres: Hurst and company.

Ranger, T. 1975 Dance and Society in Eastern Africa. Londres-Ibadan: Thèse.

Riot, T. 2010 «Le football Rwanda. Un simulacre guerrier dans la créolisation d'une société », Revue canadienne des études africaines: 75-109.

Riot, T. 2011 Sport et mouvements de jeunesse dans l'émancipation politique du Rwanda colonial. Histoire d'une libération imaginée (1935-1961). Strasbourg: Thèse de doctorat.

Riot, T. 2014 «Les politiques de "loisir" et le génocide des Tutsi rwandais. Du racisme culturel aux donjons de la mémoire (1957-2013)», Politique africaine 133: 131-151.

Taylor, C.-C. 2000 Terreur et sacrifice, une approche anthropologique du génocide rwandais. Toulouse: Octarès.

Vidal, C. 1991 Sociologie des passions (Côte d'Ivoire, Rwanda). Paris: Karthala.

Veyne, P. 1975 «Y a t-il eu un impérialisme romain?», Mélanges de l’École française de Rome-Antiquité: 793855 .

Warnier, J.-P. 2002 « Les jeux guerriers du Cameroun de l'Ouest. Quelques propos iconoclastes », Techniques ECulture 39: 177-194.

Warnier, J.-P. 2009 «Stéphane Audoin-Rouzeau, Combattre», L'Homme : 202-203.

Warnier, J.-P. 2011 «Bodily/Material Culture and the Fighter's Subjectivity», Journal of Material Culture: $1-17$.

Weigert, S. L. 2011 Angola. À Modern Military History. 1961-2002. New York: Palgrave Macmillan. 


\section{POUR CITER CET ARTICLE}

Riot, T., Bancel, N. \& H. Boistelle 2017 « Du combat. Jouer et faire la guerre en Afrique (post-)coloniale et contemporaine», in G. Bartholeyns \& F. Joulian, Le corps instrument, Techniques\&Culture 62 : 150-165.

\section{RÉSUMÉ}

Du combat. Jouer et faire la guerre en Afrique (post-) coloniale et contemporaine

«Sport is war minus fighting», disait Georges Orwell. Il proposait en ces termes de situer l'activité ludo-sportive dans le registre pratique d'un simulacre guerrier. Si l'on demeure dans le champ du simulacre (ou de la représentation sociale et symbolique), il sera difficile d'appréhender le passage du jeu au combat que nous cherchons à explorer. Ce transfert appartient à l'univers de l'agôn. Dans leur dimension agonistique, les jeux peuvent être appréhendés sous l'angle de leur hybridation avec les technologies du combat le plus concret. Dans plusieurs pays africains, l'analyse de ces pratiques révèle que le processus peut s'envisager d'une part, sous les trajectoires ludo-motrices « ordinaires» des «professionnels de la guerre» et d'autre part, sous l'angle de la compétition entre combattants d'une même armée.

\section{ABSTRACT}

\section{About Combat. Playing and Making War in (Post) Colonial and Contemporary Africa}

«Sport is war minus fighting», quoted Georges Orwell. He suggested in these terms to place leisure and sports activities within a practice register of warrior simulacrum. If we remain in the simulacrum field (or social and symbolic representation), it would be difficult to grasp the transition from play to fight, that we want to explore. This transfer belongs to agon's universe. In its agonistic dimension, plays could be understood from the standpoint of hybridization with concrete combat technology. In several African countries, the analysis of these practices exposes on one hand, «war professionals» from the perspective of « ordinary» playful-physical trajectories and on the other hand, competitions among fighters in the same army.

\section{MOTS CLÉS}

Jeux, sports, combattant, subjectivation politique, Afrique

\section{KEYWORDS}

Games, sports, fighter, political subjectivation, Africa 